\title{
Vapor Phase Cooling
}

National Cancer Institute

\section{Source}

National Cancer Institute. Vapor Phase Cooling. NCI Thesaurus. Code C78211.

A cryogenic process for the storage and/or shipping of biospecimens. The item to be frozen is placed above a shallow reservoir of cooling liquid in a storage vessel. A vertical temperature gradient is maintained throughout the vapor layer to ensure temperatures sufficiently low to maintain the integrity of the stored material. 